\title{
LXXVII. Further remarks on experiments relative to the interference of light
}

\section{Rev. Baden Powell M.A. F.R.S.}

To cite this article: Rev. Baden Powell M.A. F.R.S. (1832) LXXVII. Further remarks on experiments relative to the interference of light, Philosophical Magazine Series 3, 1:6, 433-438, DOI: $10.1080 / 14786443208647935$

To link to this article: http://dx.doi.org/10.1080/14786443208647935

册 Published online: 01 Jun 2009.

Submit your article to this journal $₫$

Џll Article views: 2

Q View related articles $₫$ 
LXXVII. Further Remarks on Experiments relative to the Interference of Light. By the Rev. BaDEN PowelL, M.A., F.R.S., Savilian Professor of Geometry, Oxford**

I $N$ a paper which appeared in the Philosophical Magazine and Ammals, N.S. for January 1832, I described a few experiments, having in view the fuller illustration of the principle of interferences, and of the undulatory theory of light. Those remarks claim no higher character than that of attempts to simplify some points in the inquiry, and facilitate the diffusion of elementary knowledge on this most beautiful and interesting branch of science; and it is with no other object that the present paper has been drawn up.

There are, in fact, few parts of science (especially considering the experimental and popular form of which it is susceptible,) which have excited less general attention than that to which the name of physical optics has been applied. And I would observe, in passing, that as the distinction implied in this designation between the properties of interference, polarization, \&c., and those of ordinary reflexion and refraction is wholly arbitrary,- these last being just as much physical properties as the former,-it would surely be better to restrict the term "physical optics" to a sense analogous to "physical astronomy," viz. to the theory of those motions and forces which shall account for the observed effects on dynamical principles. This is quite independent of the facts. Nor is the distinction merely verbal, for I believe that an imagined inseparable connexion between the facts of the coloured rings, polarization, \&c. and the physical theory, has tended in no small degree to create a reluctance in many persons to enter upon a subject which was supposed to be identified with a doubtful, abstruse, and difficult hypothesis.

In this point of view, therefore, the distinction may be by no means unimportant. Long since, indeed, those who were most profoundly versed in the subject were not backward in their attempts to dispel such misconceptions. The present Lord Chancellor Brougham, in his highly acute and original papers "On the Reflexion, Inflexion, and Colours of Light," in the Philosophical Transactions for 1796-97, insisted strenuously on the distinction between the facts of Newton's experiments (which he pursued and extended) and the theories whether of fits or waves; and though he rejected both for a simpler theory of his own, this was at a period when the real nature of either was as yet undemonstrated. And when the singular fact of interference was unequivocally established by $\mathbf{D}:$.

* Communicated by the Author.

Third Series. Vol. 1. No. 6. Dec. 1832. 


\section{Prof. Powell's Further Remarks on Experiments}

Young, it is surprising that his lectures and writings did not produce a general impression in favour of a branch of science, which his researches had successfully reduced to a form not less philosophically comprehensive, than experimentally simple and striking. More lately Fraunhofer, in his elaborate researches, has carefully pointed out the distinction that these "intervals" have a real existence, whatever theoretical view we may take of their nature. The publication of Sir J. Herschel's Treatise on Light forms an epoch in the history of the science, and has given a material impulse to the study of it: and there are only wanting further endeavours to simplify and facilitate the investigations, and to bring them more within the reach of the generality of students, in order to diffuse a knowledge of the subject as widely as its beauty and importance demand.

The only point which continued to be a matter of question, viz. the application of interferences to the colours of thin plates, having now been decisively settled by Professor Airy's experimentum crucis (Phil. Mag. and Annals, August 1831), the language of comparison between the theories which was legitimately used before, has ceased to be admissible. But the sagacity with which Newton detected the existence of these intervals, as well as the accuracy with which he measured them, only continues to be enhanced in our estimation by the more recent explanation of their nature. He observed that at certain thicknesses no sensation of light reached the eye: it was therefore a natural and unavoidable conclusion, that none was reflected,-when as yet neither the paradoxical fact that two reflexions might destroy each other, nor the equally essential point that in this case there were two reflexions concerned, had been established.

Next to the fact of the existence of the intervals, that of their diminution in more refractive media is of fundamental importance to the theory. The fact is indeed involved in a variety of phænomena: but as I have not happened to meet with any detailed account of the method of showing it experimentally, except mixed up with other considerations, I will here offer a few remarks upon the subject.

In the first place it is evident, that if we employ the inclined reflectors for the interference-stripes*, and they be immersed in a transparent fluid, the surface of the liquid medium being so arranged that the rays are incident perpendicularly, and if the eye-lens be placed in such a manner as to receive the rays

* The experiments, as well as the formula here referred to, are those described in my former paper,-Phil. Mag, and Annals, N.S. vol. xi. p. 4, \&c. 
immediately from the liquid, the stripes will be seen as formed at its focus within the liquid. This will be immediately understood on recollecting that the stripes seen by a lens, under any circumstances, are those formed at its, focus, and are to be regarded simply as an optical image situated there.

Hence also, if the lens have not its surface in contact with the liquid, but be placed at any distance from the surface less than its focal length, it will still exhibit the stripes as formed within the liquid.

In this way the stripes will be seen narrower, or having less values of $c$ than in air: and from the equation,

$$
c=\frac{\lambda}{2} \cot \theta, \text { or } \lambda=2 c \tan \theta,
$$

it follows, that since $\theta$ remains the same, $\lambda$ must be diminished within the medium. If the obtuse prism be employed, or the interference be produced by refraction, it will be manifest that, owing to the less difference in refractive power between the glass and the liquid, than between the glass and air, the rays will cross at a smaller angle; and if $\lambda$ remain the same, $c$ ought therefore to be greater; but in point of fact the stripes are observed unaltered, or $c$ remains the same: hence it follows that $\lambda$ must really be diminished. The diminution of $\theta$ is evidently due to the difference of refractive power between the liquid and air ; and if $m$ be the relative index (calling the angle in the liquid $\theta$ ), we have $\sin \theta=m \sin \theta$; and since in such very small angles we may substitute the sine for the tangent, the formula gives, in air, $\lambda=2 c \sin \theta$

$$
\text { in the liquid, } \lambda_{1}=2 c \frac{1}{m} \sin \theta,
$$

Or $\lambda_{\text {, }}$ is a value diminished exactly in the ratio of the refractive powers. Either of these experiments is, however, very troublesome to manage; and it is very difficult to compare satisfactorily the breadth of the stripes in the two cases. The following is much easier, and has the additional advantage of being applicable to solid transparent bodies as well as to liquids.

If a mass of a solid transparent body with plane parallel surfaces, or of a fluid bounded by parallel plates of glass, be simply placed between the prism or reflector and the eyelens, the latter having its focus within the medium as before, then the rays on entering the medium, as they emerge from the prism inclined to each other at an angle $2 \theta$, that angle will now be diminished to $2 \theta_{t}$, and hence, as before, the stripes formed within the medium ought to become wider; but in point of fact they are seen unaltered: they may in this case be $3 \mathrm{~K} 2$ 
easily compared with the stripes formed in air by arranging the lens so that one half of it is exposed to the direct rays, the other to those passing through the medium. The two portions of the stripes thus seen are, in fact, continued exactly in the same straight lines; and the eye would be well able to judge of the smallest deviation in such extremely delicate and well-defined lines, did any exist. The stripes, however, are precisely of the same breadth, or the values of $c$ the same, whether with or without the interposition of the medium: hence, as before, $\lambda$ must be diminished exactly in proportion as $n$ is increased. But these experiments refer only to the fact, that along the length of what we term a ray of light, there occur at intervals equal to the value of $\frac{\lambda}{2}$, points at which the ray is in some way affected with a different character, such that the concurrence of two rays at a point of like affection shall produce light, but at one of opposite affection, darkness; this character evidently changing gradually from one condition to the other. All this, however, may consist with the notion of these different affections occurring simply at successive fixed points equidistant along the ray, which are merely more crowded together in a denser medium. It is then a separate conclusion, established by distinct evidence, that these intervals are propagated along the line which represents the ray with a definite velocity, diminished in proportion to the refractive power of the medium. The simple fact of the diminution of the intervals is, however, usually presented mixed up with the consideration of this progressive propagation, or rather of its retardation in denser media, in explaining the shifting of the stripes, as in the experiments referred to in my former paper, and to which the method $I$ have suggested by means of a thin prism, offers a convenient auxiliary process; showing at once the existence of the effect and its amount. Connected with this subject, some curious researches were given by $\mathrm{Mr}$. Potter, in a paper read before the British Association for the Advancement of Science, at the Oxford meeting in June last, to the publication of which I look with great interest, especially as they were founded on my experiment just alluded to, and as their tendency appeared to be somewhat at variance with the received views on this point*.

There are many students to whom it is an important object to know what is the least possible amount of apparatus indispensable for carrying on their experiments. It may be desirable to mention, therefore, that in order to perform the

* Mr. Potter's paper here alluded to will appear in our next Number.-En. 
interference-experiments, although it is most convenient to have an apparatus like that of a solar microscope for sending the sun's rays horizontally into a dark room, and concentrating them by a small lens, whilst the mirror is capable of following the motion of the sun,-yet all these conditions are not absolutely essential. A completely darkened room is far from indispensable, and the apparatus may be perhaps more conveniently fixed in a screen, which may at any moment be placed in the sun's rays in any situation, and the effect observed at some feet distance. But the place of such an apparatus may be quite sufficiently supplied from materials everywhere at hand, by merely adjusting a common plane mirror in a proper position, and receiving the rays either through a small lens, or even a minute aperture in a screen; the sole essential being an origin of light, which is as nearly as possible an absolute point. Or, again, we may use still simpler means; for if we have only a small convex mirror, such as the bulb of a thermometer, a globule of mercury, a polished metallic button, \&c. simply placed in the sun's rays, this gives an image of the sun diminished nearly to a point; and if the light from other sources be screened, the diverging beam thus formed will suffice for showing the stripes either with the obtuse prism, or by two reflecting surfaces, not indeed with the same brilliancy and distinctness, but sufficiently to verify the facts.

This method is also successfully applicable to the fringes of edges and apertures, and the internal stripes of shadows formerly called Diffraction. And mentioning these phænomena, I may observe, by the way, that the simple property on which the explanation of these fringes is founded, viz. the tendency of light to diverge from a new origin whenever an obstacle is presented,-which is a real exception, as far as it goes, to the primary law of the rectilinear propagation of light,-does not appear to me (except, perhaps, in one passage in Sir J. Herschel's treatise, to have been placed in that prominent point of view in which it should be stated in the elementary exposition of the nature and propagation of light. 'There are also some other remarkable facts apparently dependent on it, which I am engaged in investigating.

One of the most singular experiments connected with the coloured fringes is that in which the centre of the shadow of a small circular disk appears a bright point. This experiment is difficult to perform satisfactorily; since even when such a disk is cut with the utmost care, each of the minute inequalities in its edge is magnified, and accompanied with fringes, which mix and cross in such a manner as totally to confuse the whole appearance. I have succeeded by taking up a 
small quantity of thick ink on the point of a pin, and dropping it on a clear plate of glass, by which means a sufficiently even circular edge is produced, the disk being about $\frac{1}{10}$ th inch diameter.

Oxford, Nov. 4, 1832.

LXXVIII. Account of a curious Chinese Mirror, wohich reflects from its polished Face the Figures embossed upon its Back. By Sir D. Brewster, K.H. LL.D. \& C.

$\mathrm{WE}^{\mathrm{E}}$ have just received, through the kindness of George Swinton, Esq. of Calcutta, whose zeal for the promotion of science is never relaxed, an account of a curious metallic mirror, which had been recently brought from China to Calcutta, and which was then amusing the dilettanti and perplexing the philosophers of our Eastern metropolis.

This mirror has a circular form, and is about five inches in diameter. It has a knob in the centre of the back, by which it can be held, and on the rest of the back are stamped, in relief, certain circles with a kind of Grecian border. Its polished face has that degree of convexity which gives an image of the face half its natural size; and its remarkable property is, that when you reflect the rays of the sun from the polished surface, the image of the ornamental border, and circles stamped upon the back, is seen (we presume in shadow) distinctly reflected on the wall.

The metal of which the mirror is made, appears to be what is called Chinese silver, a composition of tin and copper, like the metal for the specula of reflecting telescopes. The metal is very sonorous. The mirror has a rim of about $\frac{1}{4}$ th or $\frac{1}{6}$ th of an inch broad, and the inner part, upon which the figures are stamped, is considerably thinner.

Mr. Swinton states, that no person he has met with has either seen or heard of anything similar to this mirror. The gentleman who brought it from China, says that they are very uncommon in that country; and that this one, with a few others, was brought by a Dutch ship from Japan several years ago. On the back of one of these was a dragon, which was most distinctly reflected from the polished side. Mr. Swinton also mentions that he has seen another Chinese circular mirror, which is curiously embossed on the back. It is eight inches in diameter; but as its polish is rubbed off, he has not yet been able, by replacing it, to ascertain if it reflects a picture similar to the figures stamped upon its back. $\mathrm{Mr}$. Swinton adds, that the original mirror first described, is to be 